\title{
Clinical anatomy of human heart atria and interatrial septum - anatomical basis for interventional cardiologists and electrocardiologists. Part 2: left atrium
}

\author{
Szymon Ciuk ${ }^{1}$, Przemysław Janas ${ }^{2}$, Wiesława Klimek-Piotrowska ${ }^{2}$ \\ 'Department of General and Interventional Radiology and Neuroradiology, Wroclaw Medical University, Wroclaw, Poland \\ 2Department of Anatomy, Jagiellonian University, Medical College, Krakow, Poland
}

\section{INTRODUCTION}

Pulmonary vein (PV) isolation and linear transcatheter ablation of atrial fibrillation (AF) substrates are currently commonly performed and are effective procedures within the left atrium (LA). Moreover, new transcatheter techniques for left atrial appendage (LAA) occlusion or mitral valve repair have been developed and are now successfully used in everyday clinical practice. However, unfavourable anatomical variants of atrial structures might have a significant influence on the duration and safety of transcatheter interventions; thus, every procedure of that kind should be scrupulously planned and performed. The rapid development of such interventional procedures reveals renewed interest in heart morphology.

This comprehensive review presents a systematically detailed and clinically relevant, macroscopic morphological analysis of the LA and provides an anatomical background for the most common atrial arrhythmias and invasive cardiac procedures.

\section{LEFT ATRIUM Overview}

The LA lies in the midline, to the right and posteriorly to its respective ventricular chamber [1]. The LA is also the most posteriorly situated of the cardiac chambers. Considering the difference in the positions of the mitral and tricuspid valves, the LA is located more posterior and superior to these valves than the right atrium (RA). Anatomically, the LA consists of four components: 1) a venous part, which is a place of PV drainage; 2 ) a vestibule leading to the mitral valve (atrioventricular junction); 3) the LAA; and 4) interatrial septum, which divides it from the RA [2-4]. The LA walls can be described as ante- rior, superior, left lateral, septal (or medial), and posterior. They are different in thickness and generally appear thicker than those of the RA. The anterior wall is located behind the transverse pericardial sinus and ascending aorta. The superior wall (or the roof of the LA) lies close to the bifurcation of the pulmonary trunk and the right pulmonary artery. On the septal wall, there is no visible rim that marks the fossa ovalis, and thus the true interatrial septum cannot clearly be seen; however, significant thinning of the tissue, which corresponds to the floor of fossa ovalis, is visible. The PVs enter the LA in the posterior wall, but the location of the right ones is more inferior than the left ones. The endocardial surface of the left atrium is usually smooth, but accessory appendages such as the left atrial diverticula or tissue bridges are common findings [5]. Adjacent to the posterior wall of the LA tracheal bifurcation, the oesophagus and descending thoracic aorta are in the immediate area of the LA [6].

The mean anteroposterior diameter of the LA is $38.4 \pm 4.9 \mathrm{~mm}$ in normal patients, and it increases in patients with AF (ranging from 44.0 to $74.0 \mathrm{~mm}$ ). The left atrial volume is larger in patients with persistent $\mathrm{AF}(159.7 \pm 57.0 \mathrm{~mL})$ when compared with paroxysmal AF (129.6 $\pm 44.0 \mathrm{~mL})$ [7].

\section{Pulmonary vein ostia}

The PVs are large vessels that carry oxygenated blood from the lungs into the LA of the heart. The transition between the LA and PVs (the veno-atrial junction) is usually smooth with no folds. Thus, it is often impossible to identify the precise location of the junction when observed from the endocardial aspect of the atrium. The veno-atrial junction is usually visualised better from the epicardial aspect. 


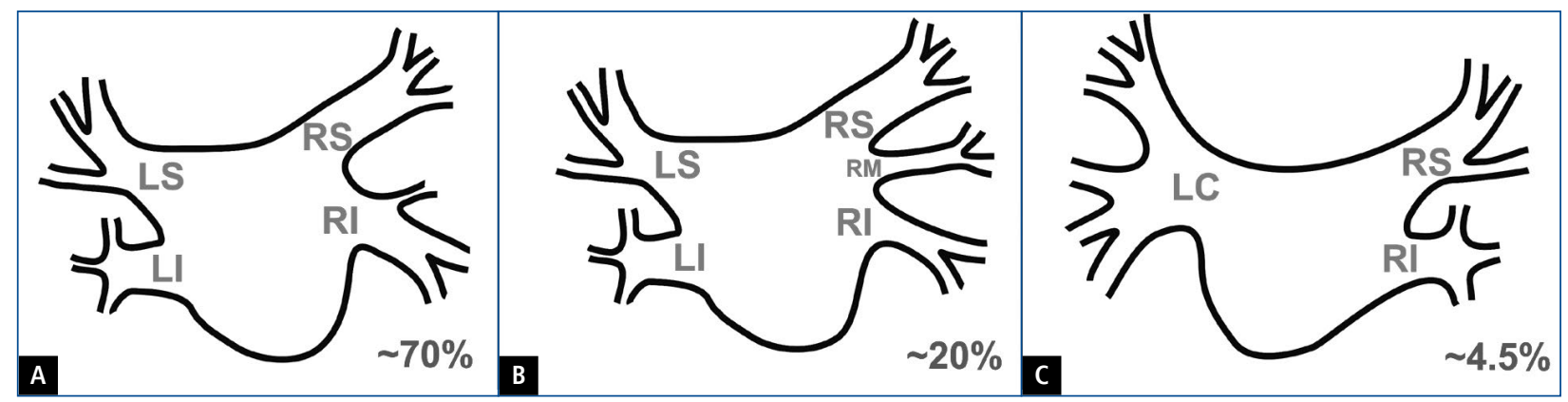

Figure 1. Schematic patterns of the most common pulmonary vein confluence variants; A. Classical pattern where single ostia of left superior (LS), left inferior (LI), right superior (RS), and right inferior (RI) pulmonary veins are observed; B. The accessory middle right pulmonary vein (RM) ostium is the second most common variant; C. Common ostium for the left pulmonary veins (LC)

The classical pattern of PV drainage is most frequently observed (in $70 \%$ of subjects) and includes two separate ostia on the left and two on the right side (the inferior and superior ones), but other anatomical variants are also observed (Fig. 1). After the classical pattern, the accessory middle right PV ostium is the second most common variation in which the middle lobe vein does not drain into the superior PV, but in approximately $20 \%$ of all patients it has an independent ostium. The third frequent anomaly consists of the common ostium for the left PVs (4.5\%). Other configurations of PV ostia are relatively rare $(<1 \%)$ and may relate to additional or common ostia (ranging from two to six ostia) [8]. The common trunk is usually very short and can be described as the antrum. Pulmonary vein ostia are ellipsoid in shape with longer superior-inferior rather than transverse dimensions. The ostial area of all veins significantly and positively correlates with LA volume [9]. Transverse ostium diameters are around $13-15 \mathrm{~mm}$ and are significantly larger for the superior PVs than for the inferior ones. When present, the additional middle right PV ostium has the smallest ostium diameter in the heart $(8.2 \pm 4.1 \mathrm{~mm})$, which is also typically too small for a cryoballoon; thus, with this method of isolation the middle right PV may easily be omitted [8]. Moreover, the right superior PV ostium diameter is significantly smaller in hearts with an additional middle right PV than in those hearts with the classical PV pattern [8]. It is disputed whether there is any association between any ostial variation and increased atrial arrhythmia; however, there is some evidence that patients with the additional right middle PV ostium and the presence of a left common PV ostium tend to present higher frequencies of AF [10].

The PV trunk is defined as the distance from the ostium to the last tributary (also known in other studies as the distance to the first bifurcation or branch). The mean length of the trunk in the cadaveric material is composed of several branches: 1) left superior PV $15.1 \pm 4.6 \mathrm{~mm}$; 2) left inferior PV $13.5 \pm 4.0 \mathrm{~mm}$; 3) right superior PV $11.8 \pm 4.0 \mathrm{~mm}$; and 4) right inferior PV $11.0 \pm 3.7 \mathrm{~mm}[8]$.

\section{Myocardial sleeves of pulmonary veins}

Despite different mechanisms of $\mathrm{AF}$, it is known that the myocardial sleeves of the PVs, especially of superior veins, are important sources of excitation leading to AF. Electrical isolation of the PV ostia has been the preferred choice for invasive treatment of symptomatic, drug-refractory, lone AF. The presence of atrial myocardial tissue extending over the wall of the PVs has been confirmed by both macroscopic and histological studies [8, 11, 12]. These myocardial sleeves are macroscopically observed in all PVs, including even the smallest additional (non-standard) ones (Fig. 2A) [8].

The transition from atrial to venous wall is gradual; in the majority of PVs (96\%) the smooth muscle cells of the venous wall overlap a layer of myocardial bundles and are only separated from them by a thin plane of fibrofatty tissue [11]. The myocardial sleeves are located between the venous media and the epicardium with adventitia and form the myocardial continuity from the LA wall with a fine matrix composed of collagen, elastic fibres, and blood vessels (Fig. 2B) [13]. The extent of the myocardial sleeves usually does not exceed the point of the last-tributary connection (10-17 mm), and the longest myocardial sleeves are found in the superior veins $[8,13]$. The thickness of the myocardial sleeve smooth muscle layer ranges from 0.05 to $1.0 \mathrm{~mm}$ at the veno-atrial junction to 0.03 to $0.5 \mathrm{~mm}$ at a distance of $10 \mathrm{~mm}$ from the junction [13]. The myocardial architecture in normal PVs is highly variable, and the sleeves are mainly composed of circularly-, obliquely-, and longitudinally-oriented bundles [13, 14]. The last orientation is suggested as one leading to anisotropic conduction between the bundles, which can act as a focal trigger for a micro re-entrant mechanism [13]. It was proven that there are gaps in the myocardial sleeves that are mainly composed of fibrous tissue and are located throughout the vein and at the veno-atrial junction. It has been suggested that they take part in AF initiation [13]. Moreover, we can find the myocardial fibres that cross the isthmus (or carina) between the superior and inferior PV ostia and thus connect the ipsilateral veins; it implies the need for isolation of this 


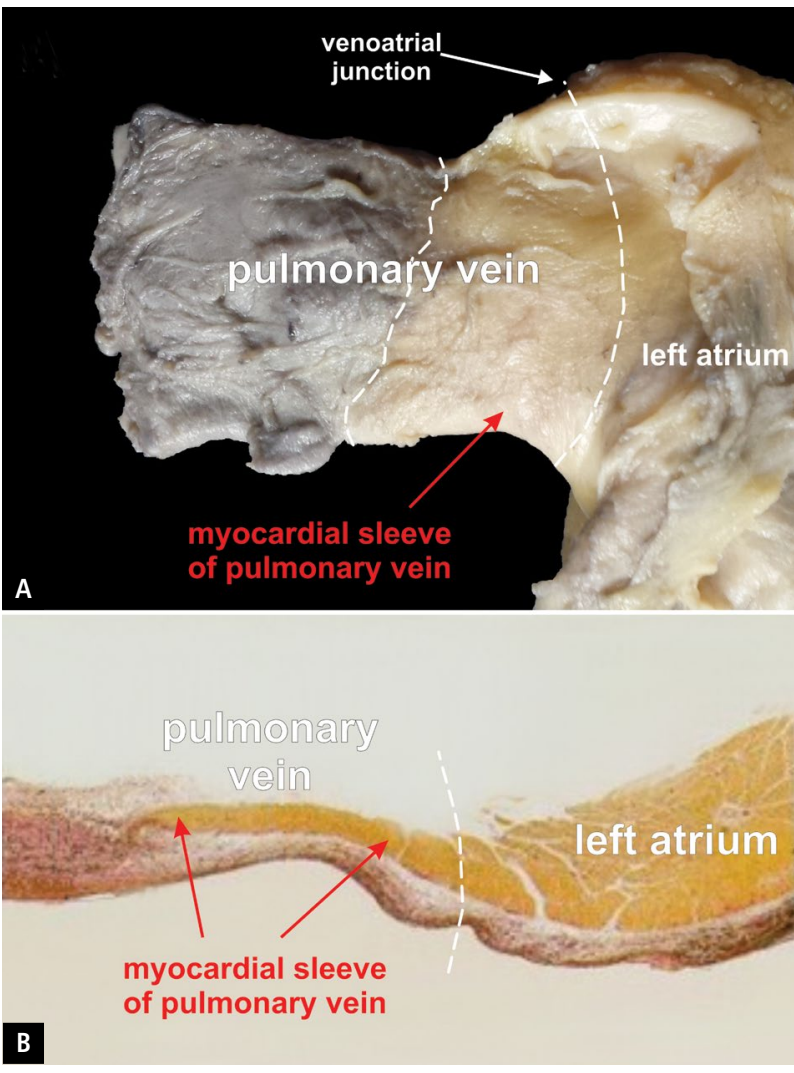

Figure 2. A. Photograph of cadaveric heart specimen. Left superior pulmonary vein with macroscopically visible myocardial sleeve; B. Microscopic appearance of myocardial sleeve. Adapted from: [8] and [14] region in order to achieve complete PV electrical disconnection $[12,15]$.

To improve the efficiency of PV isolation and prevent AF recurrence, several adjuvant lineal lesions were proposed inside the LA (Table 1).

\section{Mitral isthmus}

The mitral isthmus is a clinically defined region on the endocardial surface of the LA, which was described in 2001 by Luria et al. [16]. Based solely on clinical observations, this team proposed a new anatomical entity in the inferolateral LA of the human heart. The mitral isthmus (also called left atrial or left lateral isthmus) is part of the posteroinferior area of the lateral left atrial wall and is located between the ostium of the left inferior PV (or common left PV) and the mitral valve annulus (Fig. 3) $[17,18]$. The mean distance between the left inferior PV and the mitral annulus (mitral isthmus length) ranges from 12.6 to $59.8 \mathrm{~mm}$ (mean of $28.8 \pm 7.0 \mathrm{~mm}$ ). Regardless of the anatomical variants of the left-sided PVs, the mitral isthmus area is uniform in size [17]. The mitral isthmus line is longer than the central cavotricuspid isthmus line $(24.0 \pm 4.2 \mathrm{~mm})[19]$.

Ablation in the mitral isthmus region causes interruption of re-entrant circuits that are responsible for $\mathrm{AF}$, and it has become an attractive target for invasive electrocardiologists. Ablation within the mitral isthmus line is still the most commonly performed technique along with PV isolation, both of which result in a significant decrease in the recurrence frequency of $\mathrm{AF}$, compared to $\mathrm{PV}$ isolation

Table 1. Comparison of different endocardial lines within the left atrium that are potential targets for linear ablation

\begin{tabular}{|c|c|c|}
\hline Line & $\begin{array}{l}\text { Length }[\mathrm{mm}] \\
\text { (mean } \pm \mathrm{SD})\end{array}$ & Description \\
\hline $\begin{array}{l}\text { Mitral isthmus (or left lateral isthmus } \\
\text { or left atrial isthmus) }\end{array}$ & $28.8 \pm 7.0$ & $\begin{array}{l}\text { Located between the ostium of the left inferior PV (or common left PV) and } \\
\text { the mitral valve annulus }\end{array}$ \\
\hline Roof line & $33.3 \pm 5.3$ & $\begin{array}{l}\text { Located at the most cranial part of the left atrium and connects contralateral } \\
\text { superior PVs ostia }\end{array}$ \\
\hline Anteromedial line & $46.7 \pm 7.6$ & $\begin{array}{l}\text { The shortest line from the ostium of the right superior PV to } 10 \text { o'clock } \\
\text { position of the mitral valve annulus }\end{array}$ \\
\hline Anterolateral line & $43.9 \pm 6.2$ & $\begin{array}{l}\text { The shortest line from the medial aspect of the ostium of the left superior PV } \\
\text { to } 12 \text { o'clock position of the mitral valve annulus that did not cross the LAA } \\
\text { orifice }\end{array}$ \\
\hline $\begin{array}{l}\text { Septal isthmus (or medial left } \\
\text { atrial isthmus) }\end{array}$ & $44.5 \pm 6.3$ & Located between the ostium of right inferior PV and the mitral valve annulus \\
\hline Superolateral mitral isthmus line & $28.9 \pm 6.9$ & $\begin{array}{l}\text { Parallel to the mitral isthmus line, located immediately below the posterior } \\
\text { base of the LAA orifice and connecting left PVs with the mitral valve annulus }\end{array}$ \\
\hline Left atrial appendage isthmus & $14.2 \pm 4.8$ & $\begin{array}{l}\text { Located between the margin of the LAA orifice and the margin of the mitral } \\
\text { annulus, perpendicular to the mitral valve annulus }\end{array}$ \\
\hline
\end{tabular}

LAA — left atrial appendage; PV — pulmonary vein 
only. It is also an effective treatment option for peri-mitral flutter. However, mitral isthmus ablation can be technically challenging and may be associated with significant complications. Moreover, creating an incomplete lesion could be counterproductive and even proarrhythmogenic. Three anatomical features of this region significantly reduce the effectiveness of ablation: 1) shape and accessory structures within the isthmus line; 2) thickness of the myocardium within the isthmus; and 3) presence of the blood vessels within the mitral isthmus line.

Based on 200 heart specimens, Hołda et al. [17] proved that in $65.6 \%$ of these cases the mitral isthmus line is smooth. In the rest, different muscular and membranous structure organisational types, which were remnants of the pectinate muscles that extended from the LAA, were recorded. They can be classified into three groups: 1 ) crevices and diverticula $(18.0 \%) ; 2)$ intertrabecular recesses $(7.0 \%)$; and 3) trabecular bridges (3.5\%) (Fig. 4). In 6.0\% of the hearts, combinations of these structures were observed. Moreover, the mitral isthmus is not a flat straight line, but rather, it is concave in the majority of cases (69.5-80.0\%) or even has a pouch (4.4-20\%). In $1.5 \%$ of cases, the mitral isthmus line intersects the LAA orifice, which is interposed between the mitral annulus and left inferior PV [17]. The presence of the additional structure and shape of the mitral isthmus contributes to the diminished catheter stability and poor tissue contact and is associated with higher incidence of achieving incomplete blocks [17, 20].

The value of the tissue thickness is clinically useful because the isthmus thickness predicts ablation failure [21]. Within the mitral isthmus, the left atrial myocardium is thickest in its middle third section and thinnest in its upper third section with mean values for upper, middle, and lower one third of the mitral isthmus section of $1.9 \pm 1.0 \mathrm{~mm}, 3.0 \pm 1.5 \mathrm{~mm}$, and $2.7 \pm 1.3 \mathrm{~mm}$, respectively. In $7.5 \%$ of all hearts, a significant segmental narrowing of the myocardium in the middle section was noticed, in which the entire atrial wall was extremely thin $(<0.8 \mathrm{~mm})$, and the myocardium was composed of only a few fibres [22]. In contrast to crevices, the segmental narrowing in the middle sector of the isthmus could not be observed from the endocardial aspect. The nature of this anomaly remains uncertain, but it seems that it could be caused by impaired arrangement of the superficial layer of muscular fibres of the LA. It could be of clinical interest to evaluate the mitral isthmus anatomy before mitral isthmus ablation and, if possible, avoid the procedure in patients with extremely thin left atrial walls.

Finally, the presence of blood vessels within the isthmus line (such as left circumflex coronary artery, great cardiac vein, coronary sinus, or Marshall vein) may affect ablation in three different ways: 1) intramural blood flow in this vessel may act as an epicardial "heat sink", removing heat from the ablation site by convective cooling, thus reducing the efficacy of mitral isthmus ablation; 2) the myocardial sleeves around the coronary sinus, great cardiac, and Marshall veins may act

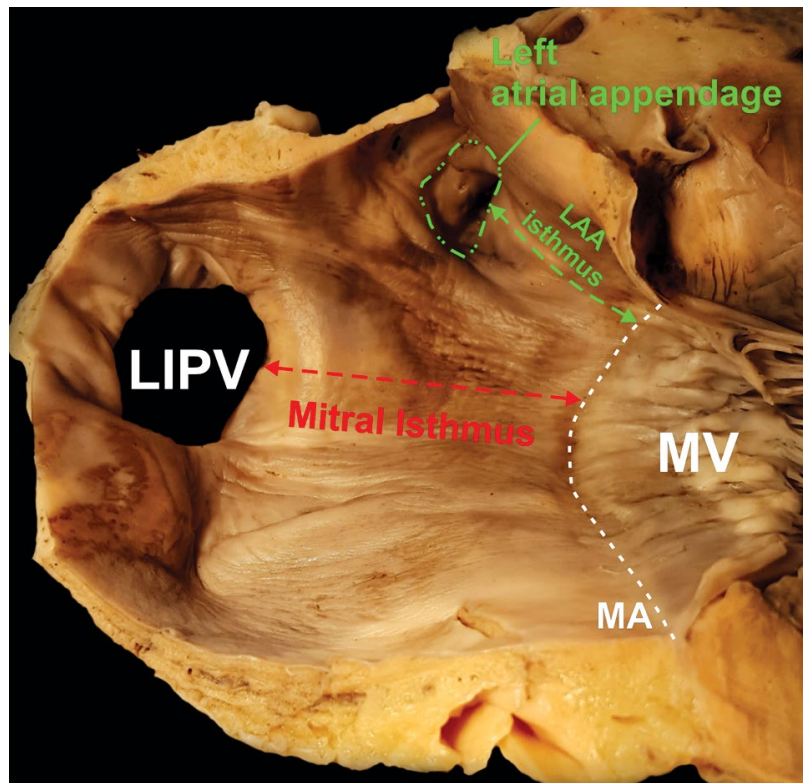

Figure 3. Photograph of a cadaveric heart specimen showing the mitral and left atrial appendage (LAA) isthmus lines. Both lines are smooth and free of any additional structures; LIPV left inferior pulmonary vein; MA — mitral annulus; MV mitral valve. Adapted from: [17]

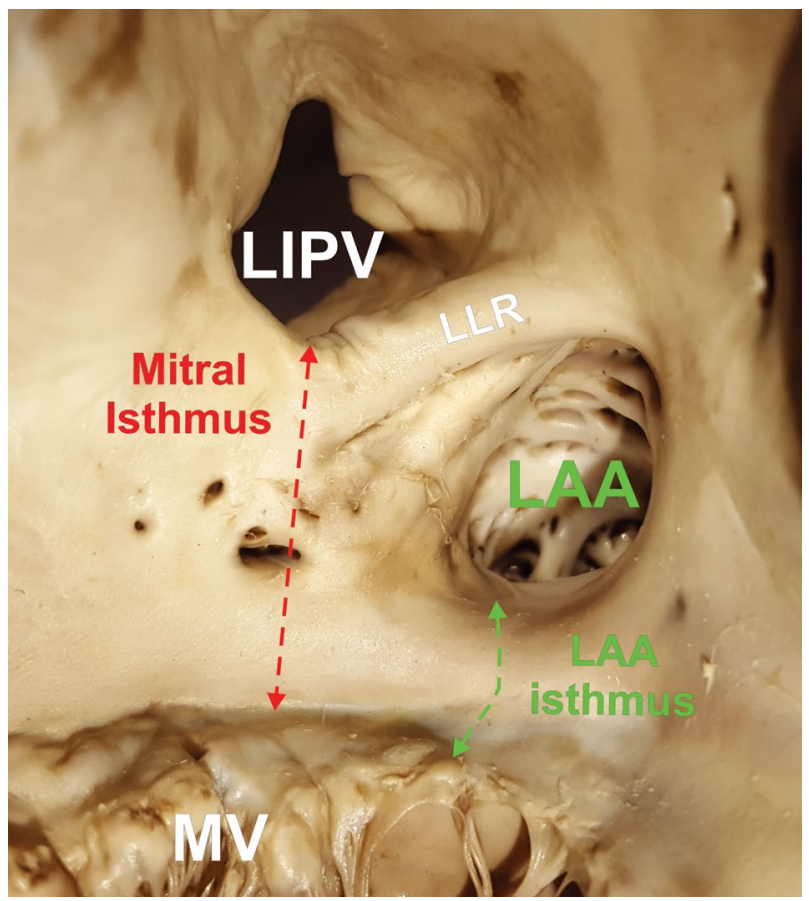

Figure 4. Photograph of a cadaveric heart specimen showing the postero-inferior area of the lateral left atrial wall. Few diverticula could be seen in the mitral isthmus area as well as disproportion between the mitral isthmus and left atrial appendage isthmus (LAA) lengths; LIPV — left inferior pulmonary vein; LLR — left lateral ridge; MV — mitral valve 


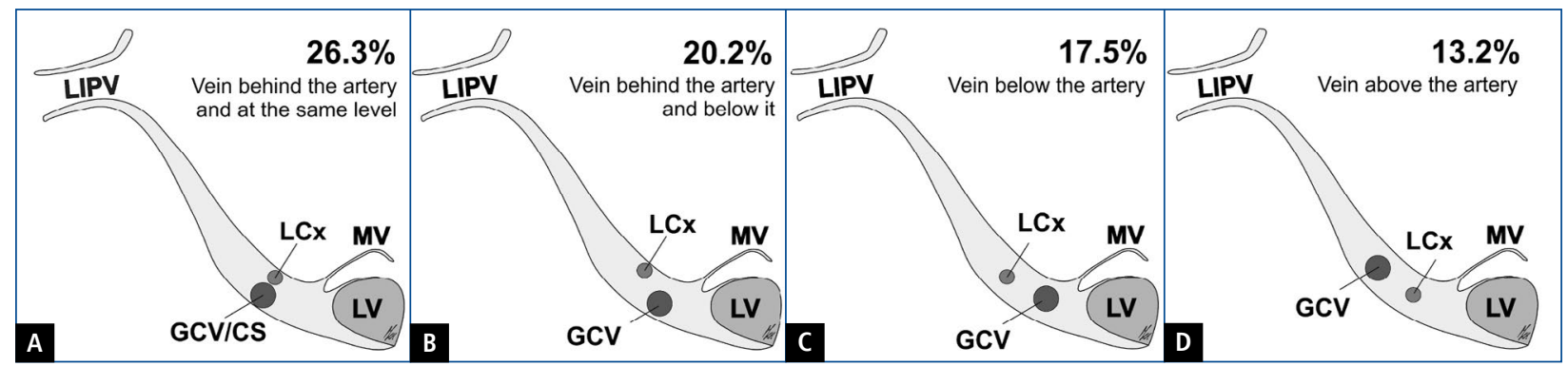

Figure 5. A-D. Schematic view of the mitral isthmus section. Four most common types of spatial relations between the great cardiac vain (GCV) and main branch of the left circumflex artery (LCX); CS - coronary sinus; LIPV — left interior pulmonary vein; LV - left ventricle; MV - mitral valve. Adapted from: [22]

as an epicardial connection that could bridge the lesion line; or 3) the close proximity of blood vessels necessitates the use of lower radiofrequency energy to avoid several complications, such as thrombosis inside the vessels, acute spasm, or even damage to blood vessel walls [23].

It was proven that the great cardiac vein is the most commonly present vessel in this region rather than the coronary sinus $(98.0 \%$ vs. $1.0 \%)$ with a mean diameter of $4.1 \pm 1.2 \mathrm{~mm}$, and it is located either in the lower one third (78.1\% of cases) or in the middle one third of the isthmus ( $21.9 \%$ of cases). The left circumflex artery is the second most commonly present vessel inside the mitral isthmus (57.0\% of hearts). The Marshall vein is present in $35.0 \%$ of all hearts and is mainly located in the middle $(69.1 \%)$ or upper (30.9\%) part of the isthmus and is the only vein to be expected in this region [22].

Not only is the presence of the particular blood vessels inside the isthmus a crucial factor influencing the ablation but also their mutual arrangement [22, 24, 25]. Both the veins and arteries within the mitral isthmus are located well above the mitral annulus. However, more blood vessels may be found closer to the lower end of the isthmus. It may be one of the explanations as to why most reconnections occur at the annular end of the ablation line. We can detect eight different patterns of mutual blood vessel arrangement within the mitral isthmus line. There are four common types: 1) vein behind the artery, but both at the same level (26.3\%); 2) vein behind and below the artery $(20.2 \%) ; 3$ ) vein below the artery but both located at the same distance from the endocardial surface (17.5\%); and 4 ) vein above the artery but both located at the same distance from the endocardial surface (13.2\%) (Fig. 5). In $16.7 \%$ of these cases, the left circumflex artery is situated at a distance $<2 \mathrm{~mm}$ from the endocardial surface, while in $49.1 \%$ it is $<3 \mathrm{~mm}$ in distance. In $55.3 \%$ of these cases, the left circumflex artery is located between the great cardiac vein and endocardial surface of the LA (interposed artery) [22]. Such interposition is a well-known predictor of unsuccessful linear ablation during endocardial and epicardial ablation [20].

\section{Alternative lines}

Pulmonary vein isolation for the treatment of AF is often supplemented with linear lesions within the LA different from the mitral isthmus line (Table 1). Alternative lines are also used for the treatment of perimitral flutter. There are conflicting data on the effectiveness of such an approach and on long-term results of additional/alternative line ablations.

The septal isthmus (or medial left atrial isthmus), which is defined as a line located between the ostium of the right inferior PV and the mitral valve annulus, was also proposed as the ablation target because of occasional involvement as part of the circuit of left atrial flutter. However, the medial isthmus was longer and consisted of more ridges than the lateral isthmus compared to the mitral isthmus, and its location close to the interatrial septum makes it difficult to ablate [26].

Recently a superolateral mitral isthmus line was proposed as a novel ablation line. It was defined as a line parallel to the mitral isthmus, located immediately below the posterior base of the LAA orifice and connecting left PVs with the mitral valve annulus. Targeting the superolateral mitral isthmus was associated with a high acute success rate in order to achieve a bidirectional block using endocardial ablation, with only a minimal need for epicardial ablation from within the coronary sinus [27].

In addition to the posterior lines that connect the PV ostia with the mitral annulus using the shortest possible pathway, several anterior lines that cross the roof of the LA and connect the superior PVs with the anterior margin of the mitral valve annulus have been suggested. Anterior lines (such as the anteromedial or anterolateral line) are significantly longer than posterior ones [28]. Moreover, they may cross Bachman's bundle and an undesired interatrial block may occur during radiofrequency ablation along anterior lines, especially the anteromedial line. Bachmann's bundle (or interatrial bundle) is a muscular bundle consisting of parallel aligned myocardial strands connecting both atria. It stretches subepicardially across the interatrial groove walls and is considered to be the main pathway of interatrial conduction [29]. 
The LAA isthmus, which is situated between the margin of the LAA orifice and the margin of the mitral annulus perpendicular to the mitral valve annulus, recently emerged as a potentially new area for ablation (Fig. 3). Hołda et al. [17] hypothesised that in some cases, the mitral isthmus may be replaced by ablation in the LAA isthmus or those two lines may be ablated together to achieve better results. It is also well known that the LAA acts as an arrhythmogenic substrate in about one-third of patients presenting for repeat ablation procedures, thus the LAA isthmus line may be useful as an additional line during LAA electrical isolation [30].

The LAA isthmus was found to be significantly shorter than the mitral isthmus (mean difference in length: $15.2 \pm 7.6 \mathrm{~mm}$; range $0.1-53.6 \mathrm{~mm}$ ). Moreover, the LAA isthmus line is smooth in $95.5 \%$ of all cases with crevices only in the remaining $4.5 \%$ [17]. As far as significant blood vessels are concerned, only left circumflex coronary arteries are expected in the direct neighbourhood of the LAA isthmus [23]. Due to the thin wall of the LAA, attention should be paid to doing ablations close to the LAA orifice. Nevertheless, the LAA walls are not very thin and are thicker than the wall inside the crevices in the mitral isthmus area. Such factors, together with the fact that the LAA isthmus is significantly shorter than the mitral isthmus, make it a prospective area for ablation procedures with potentially better long-term intervention outcomes [17].

\section{Left atrial appendage}

The LAA is a small, finger-like extension of the LA protruding from its lateral or inferolateral wall. Embryologically, it is the remnant of the original LA. The LAA is much smaller than the right atrial appendage, its length varies from 16 to $51 \mathrm{~mm}$, and it increases with age. In $80 \%$ of hearts, the LAA has a multi-lobulated appearance, and in $47 \%$ of cases sharp bends $\left(>90^{\circ}\right.$ ) of main LAA lobe are observed. The tip of the appendage can be in varied positions [31].

Because of the tubular shape of the LAA, the junction with the atrium is narrow (defined as a waist). LAA orifice superior and posterior borders are visibly demarcated by the left lateral ridge, which separates the LAA orifice from the ostia of the left PV ostia. Lacking a ridge, the LAA orifice anterior and inferior borders are not very well defined [12]. The orifice is usually elliptical or round, and in the elliptical-shaped variant, its long axis is oriented obliquely in relation to the mitral annulus. The mean long diameter of the orifice is $17.4 \pm 4.0 \mathrm{~mm}$ while its short diameter is $10.9 \pm 4.2 \mathrm{~mm}$ [32]. The shape of the LAA body varies considerably. Among several classifications that were proposed for determining the LAA shape, the simple four-step classification developed by Wang et al. [33], which divides the LAA into cauliflower (limited overall length, more complex internal characteristics, and a variable number of lobes with lack of a dominant lobe), chicken wing (with an obvious bend in the proximal or middle part of the dominant lobe, may have secondary lobes or twigs), cactus (a dominant central lobe with secondary lobes extending from the central lobe in both superior and inferior directions), or windsock (one dominant lobe of sufficient length as the primary structure), is the most common classification system. This system has potential clinical significance (Fig. 6) [33].

Left atrial appendage appears to be responsible for triggering $\mathrm{AF}$ in $27 \%$ of patients who underwent an ablation more than once. It is also a major source of cardiac thrombosis, thus significantly contributing to stroke. Several studies have demonstrated that LAA shape may play a role in its thromboembolic activity. In the autopsied material, the most common LAA type was cauliflower (41\%) followed by chicken wing (37\%), cactus (12\%), and windsock (10\%) (Fig. 6). A study, which considered the morphology of the LAA among patients with $\mathrm{AF}$, conducted using computed tomography and magnetic resonance imaging, reported different prevalence: $1)$ cactus $(30 \%) ; 2)$ chicken wing $(48 \%) ; 3)$ windsock (19\%); and 4) cauliflower (3\%) [34]. Nevertheless, assessment of LAA morphology is very difficult and should be performed only based on three-dimensional (3D) reconstructions. This may also be a cause of wide discrepancies between studies in the prevalence of the particular LAA types. However, one indisputable conclusion derived by single original studies in addition to meta-analysis indicated that patients with chicken wing LAA morphology are less likely to develop thromboembolic events than patients with non-chicken wing morphology [35].

Both the LAA itself and surrounding left atrial wall may be a target for ablation. The left circumflex artery runs epicardially in the fat-filled atrioventricular groove and close to the inferior border of the LAA orifice. This anatomical structure should always be considered when ablating inside or around the orifice of the appendage. The shortest distance from the LAA orifice to the left circumflex artery may be $<2 \mathrm{~mm}[6,17]$.

The inner LAA surface is lined with a complicated network of fine pectinate muscles $>1 \mathrm{~mm}$ thick, which makes the LAA actively contractile [12]. Considering the morphology groups, mild trabeculations are present in the chicken wing LAA morphological type, moderate trabeculations in the cactus type, and extensive trabeculations in the cauliflower type [36]. Muscular trabeculations may extend from the LAA to the vestibule of the mitral valve, but they are more commonly located in the mitral isthmus $(34.5 \%)$ than the LAA isthmus area (4.5\%) [17].

\section{Left lateral ridge}

The LA does not contain the terminal crest that divides the LAA from the body of the LA; instead, a so-called left lateral ridge, which was described in 1907 by Keith as the left tænia terminalis and later by Papez as the left posterior crest, is present at that location [37]. The left lateral ridge is the most relevant endocardial prominence of the left atrial musculature and is situated between ostia of the left-sided PVs and the LAA orifice (Fig. 7) $[10,15]$. Both the shape and size of the ridge are important fac- 


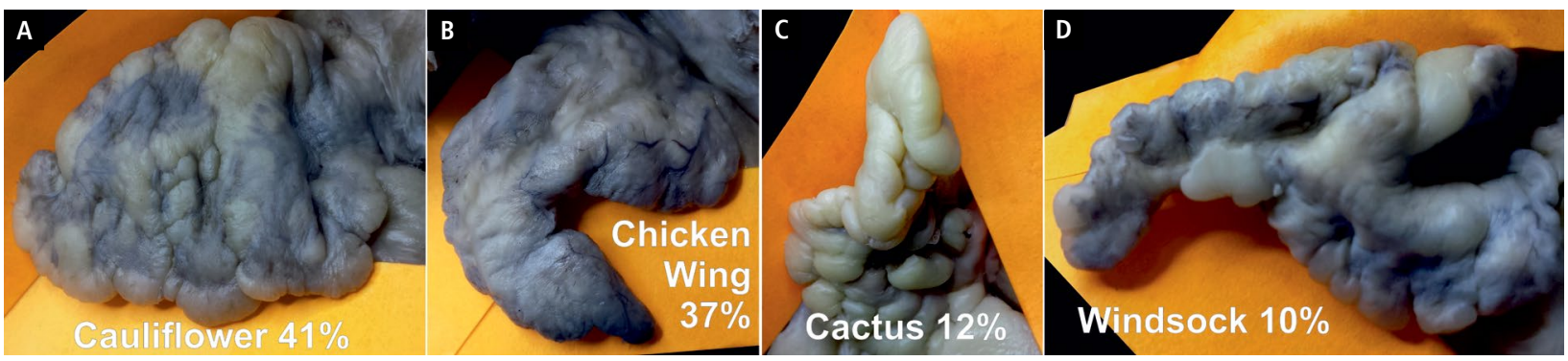

Figure 6. A-D. Photograph of a cadaveric heart specimen showing different morphological types of the left atrial appendage

tors during the ablation, which is aimed at AF treatment and is performed around the ostia of the left PVs or around/inside the LAA in the case of extrapulmonary vein triggers.

Anatomical study has demonstrated that the left lateral ridge has a narrower width and thicker myocardium superiorly rather than inferiorly [10]. Moreover, a 3D magnetic resonance study showed that the left lateral ridge is the narrowest between the right superior PV ostium and the LAA in $84 \%$ of patients (mean distance between the superior vein and the LAA and between the left inferior vein and the LAA was found to be $3.8 \pm 1.1 \mathrm{~mm}$ and $5.8 \pm 2.0 \mathrm{~mm}$, respectively). The narrow ridge is $<5 \mathrm{~mm}$ in the majority of patients and determines the possibility of obtaining a stable catheter position in this region [38]. The length of left lateral ridge varies from 14.2 to $33.5 \mathrm{~mm}$ (mean $25.3 \pm 5.5 \mathrm{~mm}$ ) with a constant superior starting point at the lateral roof of the LA extending inferiorly to the postero-inferior margin [10].

Within the left lateral ridge several structures are found: 1) the Marshall vein or ligament; 2) a small atrial artery (which in some cases is also the sinoatrial node artery); and 3) autonomic nerve bundles [15, 24, 39]. The vein or ligament of Marshall is located on the epicardial part of the left lateral ridge at a mean distance of $3 \mathrm{~mm}$ from the superior level of the ridge [15]. Myocyte bundles that cross the Marshall vein may connect the left lateral ridge with different structures such as the free wall of the LA, muscular sleeve of the coronary sinus, veno-atrial junctions of PVs, and pulmonary sleeves; this structure implies that this area is an AF trigger point [15, 40]. The artery originates mainly from the left circumflex coronary artery close to the inferior border of the LAA orifice. It runs along the epicardial part of the left lateral wall over the left lateral ridge and Marshall vein. Its mean external diameter within the ridge is $1.1 \pm 0.3 \mathrm{~mm}$ (range $0.5-1.6 \mathrm{~mm}$ ) [10]. Finally, ganglia (left ganglionated plexus) and fibres of the autonomic nervous system are present in the neighbourhood of the Marshall vein or ligament. There is a higher neural density observed in the epicardial aspect of the ridge, in particular at its superior level, in comparison to the orifice of the left superior PV. Additionally, there is an assumption that the intrinsic cardiac nerves, especially the superior left ganglionated plexus, can activate and contribute to $\operatorname{AF}[10,41]$.
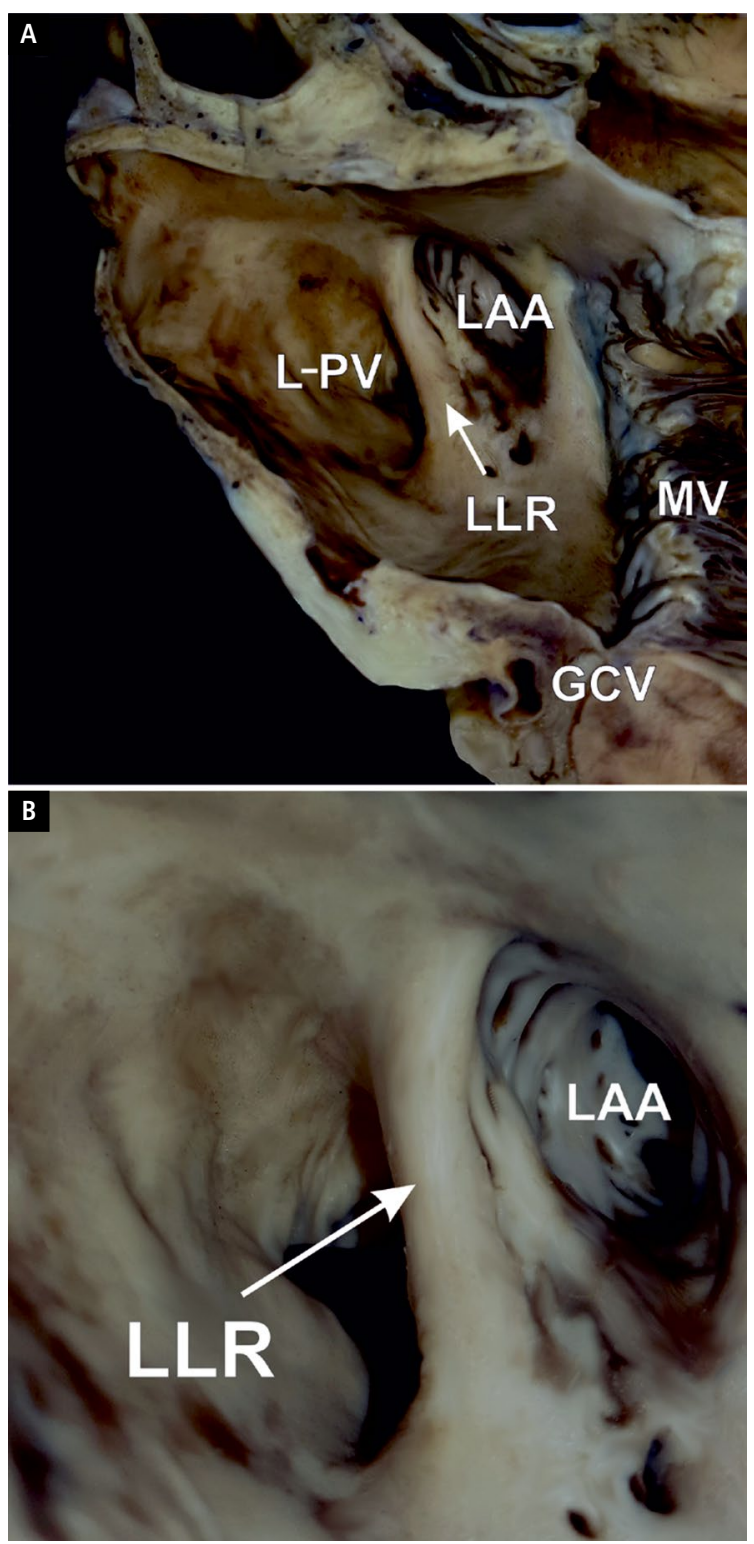

Figure 7. A, B. Photographs of a cadaveric heart specimens showing the postero-inferior area of the lateral left atrial wall with prominent left lateral ridge (LLR); GCV — great cardiac vein; L-PV — left sided pulmonary veins ostia; LAA — left atrial appendage; MV — mitral valve 


\section{Left circumflex artery and coronary sinus in} the aspect of mitral valve repair procedures

Percutaneous coronary sinus mitral annuloplasty can be an alternative treatment method for patients with ischaemic mitral regurgitation. The anatomical variants of the left circumflex coronary artery and coronary sinuous/great cardiac vein topography determine the safety of percutaneous mitral annuloplasty because the occlusion of the artery and its further consequences might occur in some cases. Therefore, knowledge of cardiac vessel anatomy is crucial. Multi-slice computed tomography makes it possible to visualise the relationship between the mitral valve annulus and surrounding blood vessels to minimise the risk of complications [42].

As mentioned above, blood vessels do not lie at the level of the mitral valve annulus but slightly above it [22]. The coronary sinus/great cardiac vein is located farthest from the annulus in the vertical plane in the middle of its course and in the horizontal plane at its beginning [43]. The artery dimensions diminish anteriorly to posteriorly, while the coronary sinus parameters increase in this direction. Blood vessels may cross each other along their whole length at different levels, and this is the case for about $63-96 \%$ of patients. The percentage of overlap in posterior and left posterior regions of the heart is rather low $(<25 \%)$, while the crossing is most commonly encountered at the anterior margin of the mitral annulus [44]. The left circumflex artery passing beneath the coronary sinus poses the highest risk of artery occlusion caused by an annuloplasty device. The left circumflex artery running closely to the mitral annulus is considered a safety feature. The most dangerous pattern occurs in cases in which two or three crossings exist between vessels [45].

\section{Left atrium surrounding structures}

There are several structures that are important in terms of a possible injury during ablation procedures. The tracheal bifurcation is located postero-superiorly to the LA with the main bronchi located superior to the right and left superior PVs. Widening of the tracheal bifurcation angle significantly correlates with the LA during its enlargement [46]. Below the tracheal bifurcation, the oesophagus has a direct relationship with the LA. In all cases, the oesophagus passes along the middle part of the posterior left atrial wall, close to the ostia of the left- rather than the right-sided PVs; however, the position of the oesophagus can vary in the posterior mediastinum, and therefore its location may be unpredictable. This proximity might result in the formation of atrio-oesophageal fistula during ablative procedures. The length of the oesophagus segment tangential to the posterior part of the LA ranges from 30.0 to $53.0 \mathrm{~mm}$ (mean $42.0 \pm 7.0 \mathrm{~mm}$ ). With atrial enlargement, the distance between the oesophagus and the left PVs may increase, but it has also high interindividual variability $[15,47]$.
Also, a layer of fibrous pericardium, which contains oesophageal arteries, the oesophageal plexus created from vagus nerve fibres, and lymph nodes, is located behind the posterior left atrial wall. The vagus nerves travel behind the root of the lungs and then form right and left posterior pulmonary plexuses. Two branches descend on the anterior surface of the oesophagus from the caudal part of the left pulmonary plexus to join the branch from the right pulmonary plexus. They form the anterior oesophageal plexus. The mean distance between the bundles of the anterior oesophageal plexus and posterior part of left atrial endocardium (or veno-atrial junctions) is estimated to be $4.1 \pm 1.4 \mathrm{~mm}$ (range $2.5-6.5 \mathrm{~mm}$ ) [48]. Thermal injury of peri-oesophageal vagal nerves during ablation procedures may result in acute pyloric spasm and gastric hypomotility.

\section{Left phrenic nerve}

Both left and right phrenic nerves may be injured during ablation procedures within the LA and PV ostia; however, thermal damage of the left phrenic nerve is less frequently observed. The right phrenic nerve is located in close proximity to the superior vena cava (minimum distance of $0.3 \mathrm{~mm}$ ) and the right superior PV (minimum distance of $2.1 \mathrm{~mm}$ ) [49].

In the lung hilum, the left phrenic nerve passes anteriorly to the left-sided PVs. It then descends on the pericardium, taking one of three courses: 1) it runs over the anterior surface of the left ventricle (in one fifth of the population); 2) over the lateral part of the left ventricle (in three fifths); and 3) in a posteroinferior direction (in one fifth) [50]. The course of the left phrenic nerve has a close relationship with the superior margin of the LAA ostium and the epicardium of the high inferolateral left ventricular wall and inferior left ventricular vein. In $31 \%$ of cases, $<2.5 \mathrm{~mm}$ separates it from the epicardium of the LAA apex. Thus, ablation within the LAA and surrounding left atrial wall can result in left phrenic nerve damage [49]. The location of the left phrenic nerve in relationship to the left atrial appendage may change with its enlargement.

\section{Conflict of interest: none declared}

\section{References}

1. Ho SY, Sánchez-Quintana D. The importance of atrial structure and fibers. Clin Anat. 2009; 22(1): 52-63, doi: 10.1002/ca.20634, indexed in Pubmed:18470938.

2. Cabrera JA, Sánchez-Quintana D. Cardiac anatomy: what the electrophysiologist needs to know. Heart. 2013; 99(6): 417-431, doi: 10.1136/heartinl-2011-301154, indexed in Pubmed: 23355600.

3. Klimek-Piotrowska W, Hołda MK, Koziej M, et al. Anatomy of the true interatrial septum for transseptal access to the left atrium. Ann Anat. 2016; 205: 60-64, doi: 10.1016/j.aanat.2016.01.009, indexed in Pubmed: 26879344.

4. Hołda MK, Koziej M, Hołda J, et al. Atrial septal pouch - Morphological features and clinical considerations. Int J Cardiol. 2016; 220: 337-342, doi:10.1016/j.ijcard.2016.06.141, indexed in Pubmed: 27390952. 
5. Cabrera JA, Ho SY, Climent V, et al. The architecture of the left lateral atrial wall: a particular anatomic region with implications for ablation of atrial fibrillation. Eur Heart J. 2008; 29(3): 356-362, doi: 10.1093/eurheartj/ehm606, indexed in Pubmed: 18245120.

6. Ho SY, Cabrera JA, Sanchez-Quintana D. Left atrial anatomy revisited. Circ Arrhythm Electrophysiol. 2012; 5(1): 220-228, doi:10.1161/CIRCEP.111.962720, indexed in Pubmed: 22334429.

7. Anselmino M, Blandino A, Beninati S, et al. Morphologic analysis of left atrial anatomy by magnetic resonance angiography in patients with atrial fibrillation: a large single center experience. J Cardiovasc Electrophysiol. 2011; 22(1): 1-7, doi: 10.1111/j.15 40-8167.2010.01853.x, indexed in Pubmed:20662985.

8. Klimek-Piotrowska W, Hołda MK, Piatek K, et al. Normal distal pulmonary vein anatomy. PeerJ. 2016; 4: e1579, doi: 10.7717/peerj.1579, indexed in Pubmed: 26793429.

9. Ratajczak P, Sławińska A, Martynowska-Rymer I, et al. Anatomical Evaluation of the Pulmonary Veins and the Left Atrium Using Computed Tomography Before Catheter Ablation: Reproducibility of Measurements. Pol J Radiol. 2016; 81: 228-232, doi: 10.12659/PJR.898650, indexed in Pubmed:27231495.

10. Marom EM, Herndon JE, Kim YH, et al. Variations in pulmonary venous drainage to the left atrium: implications for radiofrequency ablation. Radiology. 2004; 230(3): 824-829, doi: 10.1148/radiol.2303030315, indexed in Pubmed: 14739316.

11. Hassink RJ, Aretz HT, Ruskin J, et al. Morphology of atrial myocardium in human pulmonary veins: a postmortem analysis in patients with and without atrial fibrillation. J Am Coll Cardiol. 2003; 42(6): 1108-1114, doi: 10.1016/s0735-1097(03)00918-5, indexed in Pubmed: 13678939.

12. Cabrera JA, Ho SY, Climent V, et al. Morphological evidence of muscular connections between contiguous pulmonary venous orifices: relevance of the interpulmonary isthmus for catheter ablation in atrial fibrillation. Heart Rhythm. 2009; 6(8): 1192-1198, doi: 10.1016/j.hrthm.2009.04.016, indexed in Pubmed: 19632632.

13. Ho SY, Cabrera JA, Tran VH, et al. Architecture of the pulmonary veins: relevance to radiofrequency ablation. Heart. 2001; 86(3): 265-270, doi:10.1136/heart.86.3.265, indexed in Pubmed: 11514476 .

14. Saito T, Waki K, Becker AE. Left atrial myocardial extension onto pulmonary veins in humans: anatomic observations relevant for atrial arrhythmias. J Cardiovasc Electrophysiol. 2000; 11(8): 888-894, doi: 10.1111/j.1540-8167.2000.tb00068.x, indexed in Pubmed: 10969751.

15. Sánchez-Quintana D, López-Mínguez JR, Macías Y, et al. Left atrial anatomy relevant to catheter ablation. Cardiol Res Pract. 2014; 2014: 289720, doi:10.1155/2014/289720, indexed in Pubmed: 25057427.

16. Luria DM, Nemec J, Etheridge SP, et al. Intra-atrial conduction block along the mitral valve annulus during accessory pathway ablation: evidence for a left atrial. J Cardiovasc Electrophysiol. 2001; 12(7): 744-749, doi: 10.1046/j.1540-8167.2001.00744.x, indexed in Pubmed: 11469420.

17. Hołda MK, Koziej M, Hołda J, et al. Anatomic characteristics of the mitral isthmus region: The left atrial appendage isthmus as a possible ablation target. Ann Anat. 2017; 210: 103-111, doi: 10.1016/j.aanat.2016.11.011, indexed in Pubmed: 27986642.

18. Krawczyk-Ożóg A, Hołda MK, Sorysz D, et al. Morphologic variability of the mitral valve leaflets. J Thorac Cardiovasc Surg. 2017; 154(6): 1927-1935, doi:10.1016/j.jtcvs.2017.07.067, indexed in Pubmed: 28893395.

19. Klimek-Piotrowska W, Hołda MK, Koziej M, et al. Clinical Anatomy of the Cavotricuspid Isthmus and Terminal Crest. PLoS One. 2016; 11(9): e0163383, doi: 10.1371/journal.pone.0163383, indexed in Pubmed: 27682030.

20. Yokokawa M, Sundaram B, Garg A, et al. Impact of mitral isthmus anatomy on the likelihood of achieving linear block in patients undergoing catheter ablation of persistent atrial fibrillation. Heart Rhythm. 2011; 8(9): 1404-1410, doi: 10.1016/ j.hrthm.2011.04.030, indexed in Pubmed: 21699839.

21. Latcu DG, Squara F, Massaad Y, et al. Electroanatomic characteristics of the mitral isthmus associated with successful mitral isthmus ablation. Europace. 2016; 18(2): 274-280, doi: 10.1093/europace/euv097, indexed in Pubmed: 26705567.

22. Holda MK, Koziej M, Holda J, et al. Spatial relationship of blood vessels within the mitral isthmus line. Europace. 2017 [Epub ahead of print], doi:10.1093/europace/euw423, indexed in Pubmed: 28201692.

23. Wittkampf FHM, van Oosterhout MF, Loh P, et al. Where to draw the mitral isthmus line in catheter ablation of atrial fibrillation: histological analysis. Eur Heart J. 2005; 26(7): 689-695, doi: 10.1093/eurhearti/ehi095, indexed in Pubmed: 15637084.

24. Mazur M, Hołda M, Koziej M, et al. Morphology of tributaries of coronary sinus in humans - corrosion casting study. Folia Med Cracov. 2015; 55(2): 5-13, indexed in Pubmed: 26839238.

25. Tyrak KW, Holda J, Holda MK, et al. Persistent left superior vena cava. Cardiovasc J Afr. 2017; 28(3): e1-e4, doi: 10.5830/CVJA2016-084, indexed in Pubmed: 28759082.

26. Chiang SJ, Tsao HM, Wu MH, et al. Anatomic characteristics of the left atrial isthmus in patients with atrial fibrillation: lessons from computed tomographic images. J Cardiovasc Electrophysiol. 2006; 17(12): 1274-1278, doi: 10.1111/j.1540-8167.2006.00645.x, indexed in Pubmed: 17096659.

27. Maurer T, Metzner A, Ho SY, et al. Catheter Ablation of the Superolateral Mitral Isthmus Line: A Novel Approach to Reduce the Need for Epicardial Ablation. Circ Arrhythm Electrophysiol. 2017; 10(10), doi: 10.1161/CIRCEP.117.005191, indexed in Pubmed: 29018165.

28. Cho Y, Lee W, Park EA, et al. The anatomical characteristics of three different endocardial lines in the left atrium: evaluation by computed tomography prior to mitral isthmus block attempt. Europace. 2012; 14(8): 1104-1111, doi: 10.1093/europace/eus051, indexed in Pubmed: 22417722.

29. van Campenhout MJH, Yaksh A, Kik C, et al. Bachmann's bundle: a key player in the development of atrial fibrillation? Circ Arrhythm Electrophysiol. 2013; 6(5): 1041-1046, doi: 10.1161/CIRCEP.113.000758, indexed in Pubmed: 24129206.

30. Panikker S, Jarman JWE, Virmani R, et al. Left Atrial Appendage Electrical Isolation and Concomitant Device Occlusion to Treat Persistent Atrial Fibrillation: A First-in-Human Safety, Feasibility, and Efficacy Study. Circ Arrhythm Electrophysiol. 2016; 9(7), doi: 10.1161/CIRCEP.115.003710, indexed in Pubmed: 27406602.

31. Qamruddin S, Shinbane J, Shriki J, et al. Left atrial appendage: structure, function, imaging modalities and therapeutic options. Expert Rev Cardiovasc Ther. 2010; 8(1): 65-75, doi: 10.1586/erc.09.161, indexed in Pubmed: 20014936.

32. Su P, McCarthy KP, Ho SY. Occluding the left atrial appendage: anatomical considerations. Heart. 2008; 94(9): 1166-1170, doi: 10.1136/hrt.2006.111989, indexed in Pubmed: 17488765.

33. Wang Y, Di Biase L, Horton RP, et al. Left atrial appendage studied by computed tomography to help planning for appendage closure device placement. J Cardiovasc Electrophysiol. 2010; 21(9): 973-982, doi: 10.1111/j.1540-8167.2010.01814.x, indexed in Pubmed: 20550614.

34. Di Biase L, Santangeli P, Anselmino M, et al. Does the left atrial appendage morphology correlate with the risk of stroke in patients with atrial fibrillation? Results from a multicenter study. J Am Coll Cardiol. 2012; 60(6): 531-538, doi: 10.1016/j. jacc.2012.04.032, indexed in Pubmed: 22858289

35. Lupercio F, Carlos Ruiz J, Briceno DF, et al. Left atrial appendage morphology assessment for risk stratification of embolic stroke in patients with atrial fibrillation: A meta-analysis. Heart Rhythm. 2016; 13(7): 1402-1409, doi: 10.1016/j.hrthm.2016.03.042, indexed in Pubmed: 27016474. 
36. Anselmino M, Scaglione M, Di Biase L, et al. Left atrial appendage morphology and silent cerebral ischemia in patients with atrial fibrillation. Heart Rhythm. 2014; 11(1): 2-7, doi: 10.1016/j. hrthm.2013.10.020, indexed in Pubmed: 24120872.

37. Papez J. Heart musculature of the atria. Am J Anat. 1920; 27(3) 255-285, doi: 10.1002/aja.1000270302.

38. Mansour M, Refaat M, Heist EK, et al. Three-dimensional anatomy of the left atrium by magnetic resonance angiography: implications for catheter ablation for atrial fibrillation. J Cardiovasc Electrophysiol. 2006; 17(7): 719-723, doi: 10.1111/j.15408167.2006.00491.x, indexed in Pubmed: 16836666.

39. Holda M. A left circumflex artery to right atrium fistula supplying the sinoatrial node. Turkish J Thorac Cardiovasc Surg. 2016; 24(1): 155-157, doi:10.5606/tgkdc.dergisi.2016.11665.

40. Hwang C, Wu TJ, Doshi RN, et al. Vein of marshall cannulation for the analysis of electrical activity in patients with focal atrial fibrillation. Circulation. 2000; 101(13): 1503-1505, doi: 10.1161/01. cir.101.13.1503, indexed in Pubmed: 10747341.

41. Choi EK, Shen MJ, Han S, et al. Intrinsic cardiac nerve activity and paroxysmal atrial tachyarrhythmia in ambulatory dogs. Circulation. 2010; 121(24): 2615-2623, doi: 10.1161/CIRCULATIONAHA.109.919829, indexed in Pubmed: 20529998.

42. Gopal A, Shah A, Shareghi S, et al. The role of cardiovascular computed tomographic angiography for coronary sinus mitral annuloplasty. J Invasive Cardiol. 2010; 22(2): 67-73, indexed in Pubmed: 20124591.

43. Randhawa A, Saini A, Aggarwal A, et al. Spatial relationship of coronary sinus-great cardiac vein to mitral valve annulus and left circumflex coronary artery: implications for cardiovascular interventional procedures. Cardiovasc Pathol. 2016; 25(5): 375-380, doi: 10.1016/j.carpath.2016.06.001, indexed in Pubmed: 27318127.
44. Spencer JH, Prahl G, Iaizzo PA. The prevalence of coronary sinus and left circumflex artery overlap in relation to the mitral valve. J Interv Cardiol. 2014; 27(3): 308-316, doi: 10.1111/joic.12106, indexed in Pubmed: 24617351.

45. Młynarski R, Młynarska A, Sosnowski M. Anatomical variants of left circumflex artery, coronary sinus and mitral valve can determine safety of percutaneous mitral annuloplasty. Cardiol J. 2013; 20(3): 235-240, doi: 10.5603/CJ.2013.0067, indexed in Pubmed: 23788296.

46. Murray JG, Brown AL, Anagnostou EA, et al. Widening of the tracheal bifurcation on chest radiographs: value as a sign of left atrial enlargement. AJR Am J Roentgenol. 1995; 164(5): 1089-1092, doi: 10.2214/ajr.164.5.7717208, indexed in Pubmed: 7717208.

47. Cury RC, Abbara S, Schmidt S, et al. Relationship of the esophagus and aorta to the left atrium and pulmonary veins: implications for catheter ablation of atrial fibrillation. Heart Rhythm. 2005; 2(12): 1317-1323, doi: 10.1016/j.hrthm.2005.09.012, indexed in Pubmed: 16360083.

48. Ho SY, Cabrera JA, Sánchez-Quintana D. Vagaries of the vagus nerve: relevance to ablationists. J Cardiovasc Electrophysiol. 2006; 17(3): 330-331, doi:10.1111/j.1540-8167.2006.00364.x, indexed in Pubmed: 16643412.

49. Sánchez-Quintana D, Cabrera JA, Climent V, et al. How close are the phrenic nerves to cardiac structures? Implications for cardiac interventionalists. J Cardiovasc Electrophysiol. 2005; 16(3): 309-313, doi: 10.1046/j.1540-8167.2005.40759.x, indexed in Pubmed: 15817092.

50. Sánchez-Quintana D, Ho SY, Climent V, et al. Anatomic evaluation of the left phrenic nerve relevant to epicardial and endocardial catheter ablation: implications for phrenic nerve injury. Heart Rhythm. 2009; 6(6): 764-768, doi: 10.1016/j.hrthm.2009.02.029, indexed in Pubmed: 19427272.

Cite this article as: Ciuk S, Janas P, Klimek-Piotrowska W. Clinical anatomy of human heart atria and interatrial septum — anatomical basis for interventional cardiologists and electrocardiologists. Part 2: left atrium. Kardiol Pol. 2018; 76(3): 510-519, doi: 10.5603/KP.a2018.0001. 